\title{
A History of Kawasaki Disease From Childhood and Coronary Artery Ectasia With Recurrent ST Elevation Myocardial Infarction: A Therapeutic Challenge
}

\author{
Ashraf Abugroun a, b, Daniel Vilchez ${ }^{\mathrm{a}}$, Osama Hallak ${ }^{\mathrm{a}}$, \\ Anahita Shahrrava $^{\mathrm{a}}$
}

\begin{abstract}
Coronary artery ectasia (CAE) is an uncommon anomaly, usually found in $1.2-2 \%$ of patients undergoing coronary angiography, defined as a segment of the coronary artery that has a diameter of more than 1.5 times the normal adjacent segments. Atherosclerosis is considered as the cause of half of the CAE cases. We herein present a 65-year-old Asian male with past medical history of Kawasaki disease (KD) who developed recurrent episodes of inferior wall STelevation myocardial infarction (STEMI) despite treatment with dual antiplatelet therapy (DAPT). Repeat coronary angiogram showed severely ectatic and tortuous coronary arteries more predominant on the right coronary artery (RCA) with diffuse thrombus in its mid segment. Given his unfavorable vascular anatomy, the condition was managed medically with the addition of warfarin to his DAPT with target international normalized ratio (INR) 2 - 3. This case highlights the association of CAE with a prior history of $\mathrm{KD}$ and its therapeutic challenge.
\end{abstract}

Keywords: Kawasaki disease; Coronary artery ectasia; Myocardial infarction

\section{Introduction}

Kawasaki disease (KD, mucocutaneous lymph node syndrome) is an acquired cause of medium sized vasculitis of unknown etiology that commonly affects the coronaries. While the disease is predominantly seen among children, particularly of Asian descent, chronic vascular sequela including diffuse coronary vascular ectasia remains a therapeutic challenge. Coronary artery ectasia (CAE) is an uncommon anomaly, usually found in $1.2-2 \%$ of patients undergoing coronary angiog-

Manuscript submitted November 22, 2017, accepted December 8, 2017

aAdvocate Illinois Masonic Medical Center, Chicago, IL 60657, USA

${ }^{b}$ Corresponding Author: Ashraf Abugroun, Department of Internal Medicine, Advocate Illinois Masonic Medical Center, 836 W Wellington Ave, Chicago, IL 60657, USA. Email: ashraf.abugroun@advocatehealth.com

doi: https://doi.org/10.14740/cr641w raphy, defined as a segment of the coronary artery that has a diameter of more than 1.5 times the normal adjacent segments [1]. Atherosclerosis is considered as the cause of half of the CAE cases. This case highlights the association of CAE with a prior history of $\mathrm{KD}$ and its therapeutic challenge.

\section{Case Report}

A 65-year-old Asian male with past medical history of KD, hypertension, and hyperlipidemia, came for follow-up in the cardiology clinic following a recent hospital admission for inferior wall ST-elevation myocardial infarction (STEMI) in 2016.

The patient's history of KD started as early as year 1956 when he was 6 years old. He developed an abnormal clinical symptom-complex characterized with fever and rash. None of his siblings developed similar condition. His condition was mistakenly diagnosed as scarlet fever as during that time KD was not yet described. The patient was informed several years later, that his childhood illness was actually $\mathrm{KD}$. He was not on treatment for the condition.

His first myocardial infarction (MI) was in 2009, when he was diagnosed with an inferior wall STEMI (Fig. 1). Coronary angiogram revealed diffuse ectatic changes of all coronaries with no suitable lesion identified for angioplasty or stenting, therefore he was medically managed with dual antiplatelet therapy (DAPT) with aspirin and clopidogrel. His vascular lesions were typical for chronic Kawasaki vascular disease. Following discharge, he developed palpitations and frequent unifocal premature ventricular contractions (PVCs) exceeding 10,000 per $24 \mathrm{~h}$. These symptoms were adequately controlled with metoprolol.

Echocardiography done 3 years later showed a left ventricular (LV) ejection fraction of $45 \%$. Patient underwent a stress echo that showed stable inferior-posterior wall motion abnormality without new ischemic changes, accordingly the drop of LVEF was attributed to PVC mediated myopathy. Despite the high PVC burden, metoprolol adequately controlled his symptoms and his LV function remained stable for several years. The patient followed a healthy life style, exercised regularly, and never used any tobacco products. He had a normal physical exam with body mass index (BMI) of 27. Despite these healthy measures and compliance with optimum medi- 


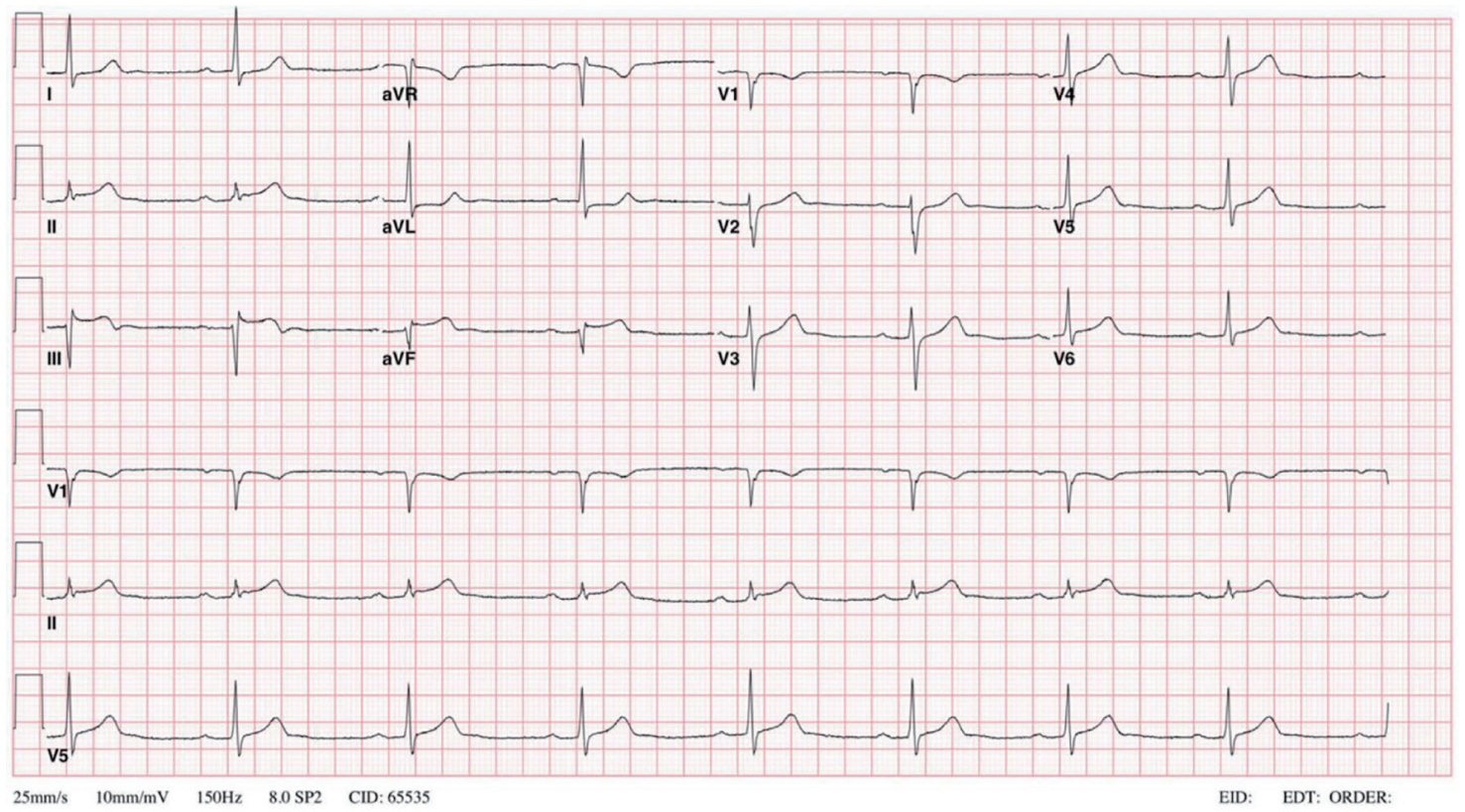

Figure 1. EKG done on 2009 showing sinus bradycardia, first degree AV block and ST segment elevations $>0.1 \mathrm{mV}$ in the inferior leads II, III, and aVF. EKG: electrocardiogram; AV: atrioventricular.

cal therapy, he developed acute inferior wall STEMI on April 2016, followed by another attack by the end of the same year (Fig. 2). Coronary angiogram showed severely ectatic and tortuous coronary arteries (Fig. 3) more predominant in the right coronary artery (RCA) with a diffuse thrombus in its mid segment (Fig. 4). Given his unfavorable vascular anatomy, his condition was managed medically with the addition of warfarin to his DAPT with target international normalized ratio
(INR) 2 - 3.

\section{Discussion}

$\mathrm{KD}$ is characterized by an acute necrotizing vasculitis of the medium- and small-sized vessels. It is most prevalent in Japan, followed by Korea, predominantly seen in children aged

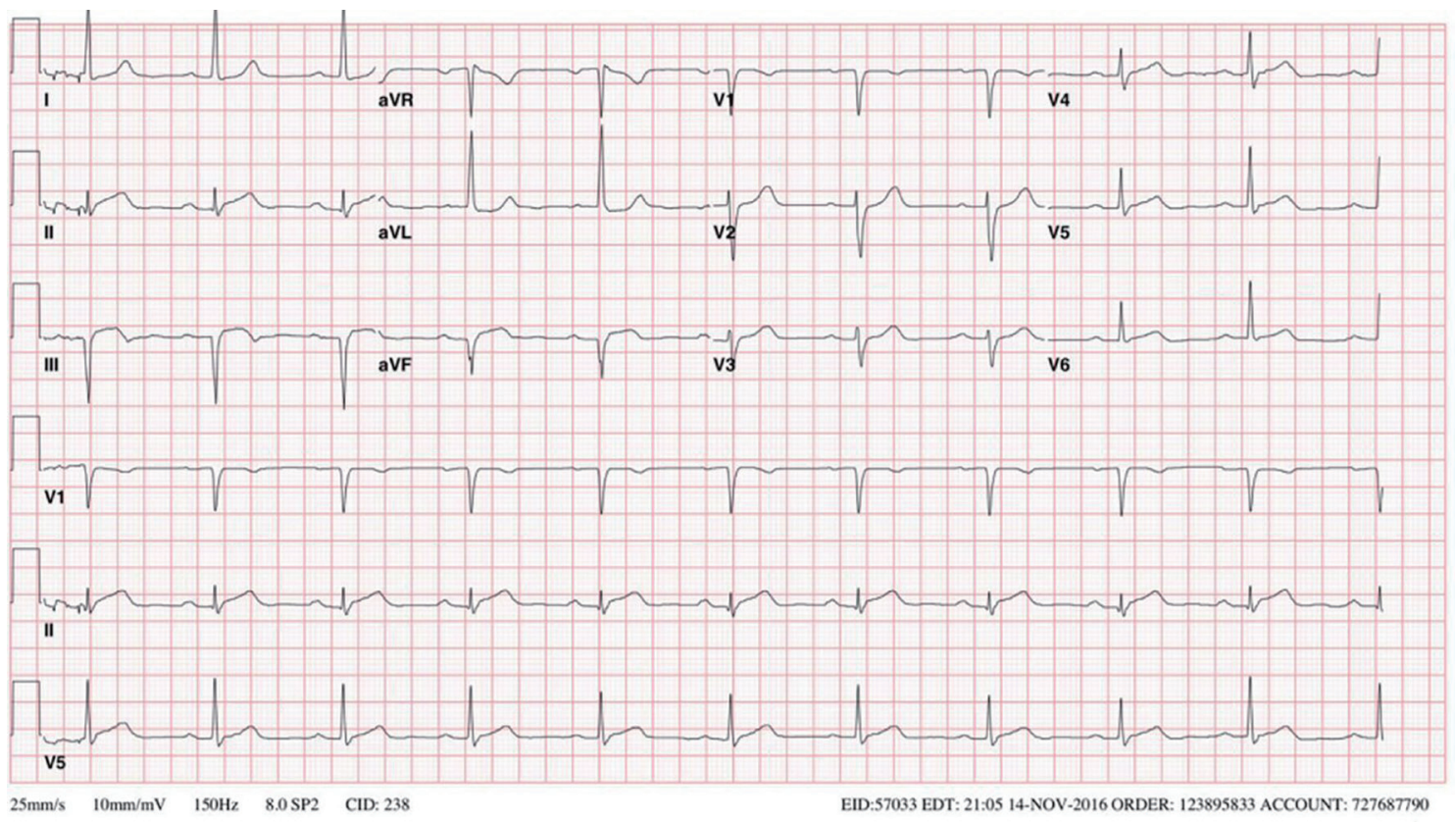

Figure 2. EKG on late 2016 showing sinus rhythm with a prolonged PR interval, left ventricular hypertrophy based on the height of the $\mathrm{R}$ wave in $\mathrm{aVL}$ and an inferior infarct based on ST elevations in leads II, III, and aVF. 


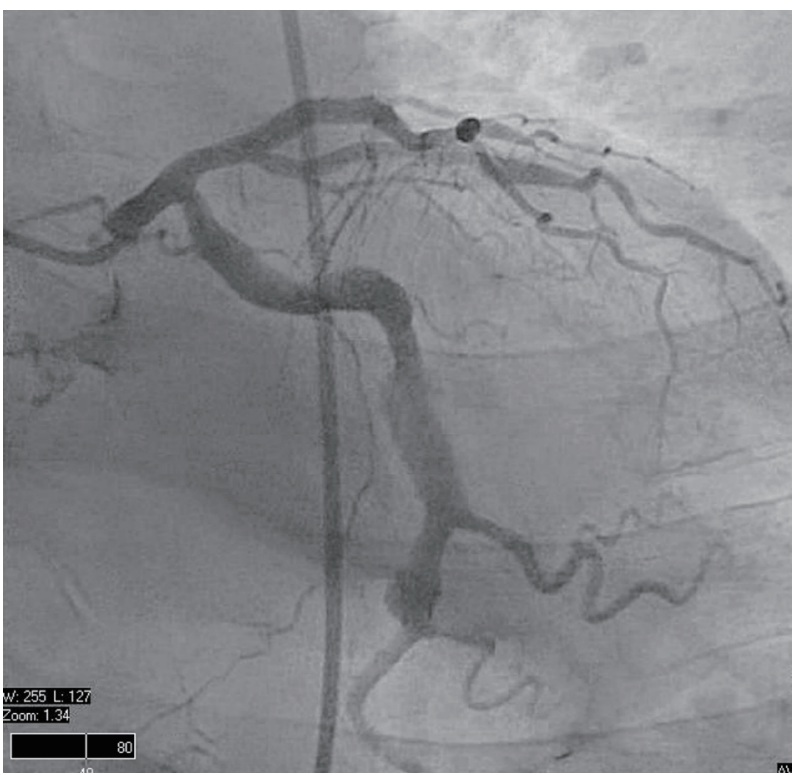

Figure 3. RAO caudal view of left coronary system. Left circumflex artery is a large ectactic vessel. RAO: right anterior oblique.

from 6 months to 5 years, and has a male-to-female ratio of $1.5-1.8$. The first report of KD was published in 1967. During that time, KD was described as a self-limiting disease without sequela. It wasn't until 1970 when the association between KD and coronary artery vasculitis was well-established [2]. The etiopathogenesis of the condition is not fully understood, but it is likely multifactorial with possible role of various environmental, infectious, and genetic factors. KD is the most severe vasculitic syndrome affecting children younger than age 5 and has now surpassed rheumatic fever to become the leading cause of acquired heart disease in children living in developed countries $[3,4]$.

$\mathrm{KD}$ is suspected when a patient develops marked coronary artery ectasia or aneurysm formation despite young age and minimum risk factors for atherosclerotic coronary artery disease [5].

There are few reports in the literature discussing the occurrence of recurrent myocardial infarctions among patients with coexisting CAE. By reporting this case, we would like to highlight an important therapeutic challenge of CAE. We will cover the current proposed pathophysiology of CAE as well as the available diagnostic and therapeutic options.

The characteristic hallmark of KD is the presence of coronary artery damage. Other complications related to KD include pericardial effusion, myocarditis, valvular dysfunction, and acquired aneurysms of the systemic arteries. Coronary aneurysms, defined as dilation of the coronary artery to a diameter of at least 1.5 times that of normal adjacent segment, caused by KD have variable outcomes. Small lesions are likely to resolve, while larger lesions have potential for a wide range of remodeling changes [6]. Aneurysmal dilation of the coronary can be spherical or saccular in shape, and either diffuse, affecting the entire length of the vessel, or localized. The diffuse form of the disease is referred to as ectasia.

$\mathrm{CAE}$ is found in up to $5 \%$ of patients undergoing coronary

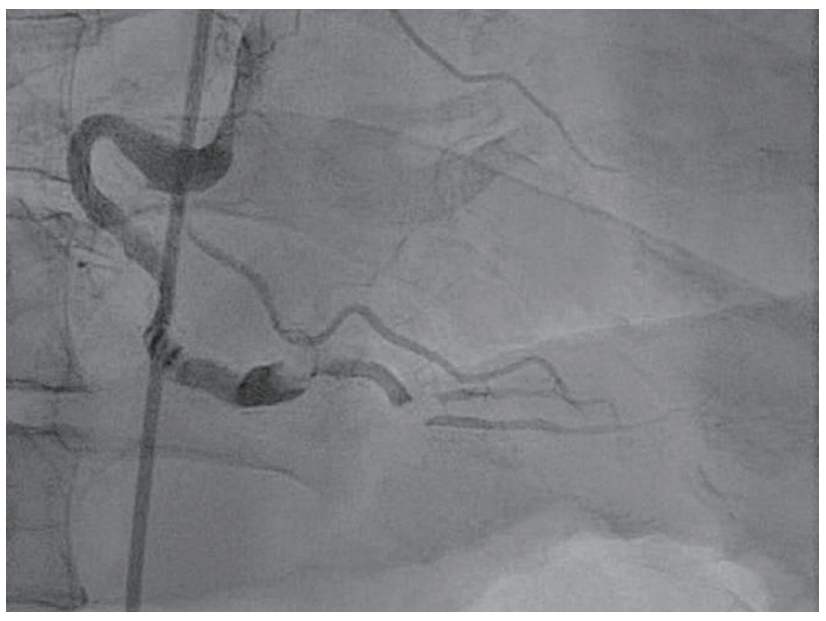

Figure 4. RAO cranial view of right coronary system. RCA is a severe ectactic vessel with diffuse thrombus in mid-segment. RCA: right coronary artery.

angiography and in $0.22 \%$ to $1.4 \%$ of autopsy series [7]. CAE commonly coexists with coronary artery disease (CAD). The pathogenesis of CAD induced CAE is not fully understood. It includes an initial endothelial damage that activates a series of inflammatory changes causing degeneration of the medial layer and remodeling of the vessel [8]. Recent researches have revealed a key role of proteases and the inflammatory system in the pathogenesis of CAE. The 5A/5A genotype of MMP-3 was found to be significantly more frequent in patients with CAE compared to those with atherosclerotic heart disease (ASHD) without CAE. Lamblin et al proposed that this increase in proteolysis of the arterial wall may be a susceptibility factor for the development of CAE [9]. Overexpression of matrix metalloproteinases (MMPs) leads to increased proteolysis of extracellular matrix proteins, and concurrent decreased levels of tissue inhibitors of metalloproteinases (TIMPs) strengthen this effect [1]. Others found increased levels of MMP-3, MMP-9, and IL-6 in patients with CAE compared to patients with ASHD and controls with normal coronaries undergoing catheterization. Plasma levels of adhesion molecules such as E-selectin, intercellular adhesion molecule-1 (ICAM-1), and vascular adhesion molecule-1 (VCAM-1) were also found to be higher in CAE patients, likely due to their role in facilitating the action of immune cells in the inflammatory response [1].

Upon histopathologic evaluation of ectatic arteries, the findings are similar to those in atherosclerotic arteries, including lipid deposition, degradation of medial collagen and elastin fibers, and disruption of internal and external elastic lamina [1]. Sorrell et al suggested that chronic overstimulation of the endothelium by nitric oxide (NO) or NO donors linked between atherosclerosis and CAE [10], whereas an opposing opinion claims that CAE is not truly a variant of atherosclerosis based on studies showing no difference in traditional cardiovascular risk factors of patients with pure CAE and those with CAE and atherosclerosis, except smoking which was more prevalent in the mixed group. Some studies have found a strong correlation between CAE and aortic aneurysms, with angiotensin II, which has a negative effect on vascular wall function and remodeling, 
being the link between them [1]. This brings us to the theory that increased MMP activity and decreased TIMPs lead to increased proteolysis of extracellular matrix proteins, followed by disruption of the internal elastic lamina as a gateway for inflammatory cells, as well as expansive arterial remodeling, finally leading to the development of coronary artery ectasia.

All three coronary vessels can be affected by CAE, but in the majority of patients, only a single artery is involved. The most common locations for aneurysmal formation in patients with concomitant $\mathrm{CAD}$ are the proximal and mid segments of the RCA, followed by the left anterior descending artery (LAD) and left circumflex (LCX), and least frequently reported along the left main coronary artery [11]. The reason for the higher rates of RCA involvement by CAE is not fully understood. The most common etiology of aneurysmal dilation in adults is atherosclerosis accounting for $50 \%$ of cases. In the remainder of cases, it could be congenital or acquired, secondary to inflammatory, infectious diseases, connective tissue diseases, or following invasive coronary interventions and rarely, trauma [7].

Acute coronary syndrome in patients with coronary artery aneurysm is commonly caused by plaque rupture. It can also be caused by sluggish or turbulent blood flow in the ectatic vessel which results in thrombus formation and distal embolization. Other possible complications include vasospasm and vascular wall rupture $[7,12,13]$. Measurement of thrombosis in myocardial infarction (TIMI) frame count helps in evaluation of coronary blood flow. Decreased TIMI frame count correlates with increased ectasia ration and suggests a worse prognosis in patients with CEA $[14,15]$. Ectatic vessels have higher rates for structural alterations and TIMI frame count which contribute to the increased risk for thrombosis and vasospasm. This could also worsen by blood reflux due to possible absence of diastolic antegrade flow gradient due to coronary dilation [16].

The diagnosis of CAE is mainly achieved by X-ray coronary angiography. Characteristic angiographic findings that are suggestive of KD include marked proximal ectasia followed by sudden transition to an angiographically normal distal segment. Another characteristic feature is the presence of calcification of the arterial wall along sites where former aneurysms have remodeled [5]. Better assessment of luminal size and arterial wall changes could be achieved by intravascular ultrasound (IVUS), which helps in evaluation of the severity of intimal hyperplasia, detection of thrombi, calcification, and severity of luminal narrowing [17]. Other diagnostic tests include three-dimensional, non-contrast-enhanced, freebreathing coronary magnetic resonance angiography (MRA) and coronary artery computed tomography (CACT) [7] .

The medical management of CAE is not adequately addressed. The goals of therapy in $\mathrm{KD}$ are to control and manage the cardiovascular risk factors that can contribute to disease progression and to administer medications that can improve disease prognosis. According to the Japanese Circulation Society for management of adult $\mathrm{KD}$, patients with $\mathrm{KD}$ who develop angina or MI are treated in a similar way to patients with such conditions due to other causes [17].

Among patients with coronary aneurysms, available therapeutic options include anti-platelet agents such as aspirin, as well as anticoagulant therapy for patients with giant aneurysms or thrombi in their coronary aneurysms [18]. However, these treatment options have not been tested prospectively on wide scale studies. Patients with stenotic coronary artery lesions may benefit from the use of anti-spasmic agents, such as calcium channel blockers (CCB) and nitrates [19], as well as $\beta$-blockers, angiotensin-converting enzyme (ACE) inhibitors, and/or angiotensin receptor II blockers for treatment of associated heart failure. However, the use of vasodilating agents, including nitrates, among patients with CAE is discouraged as medication induced coronary vasodilation can result in worsening of MI. Due to the close relationship between CAE and $\mathrm{CAD}$, intense cardiovascular risk factor modification is necessary. ACS caused by acute thrombotic occlusion in patients with CAE can be treated with heparin infusion and fibrinolysis. For patients with coexisting obstructive lesions with ongoing ischemia despite medical therapy, available therapeutic options include percutaneous and/or surgical coronary vascularization. Coronary artery bypass grafting $(\mathrm{CABG})$, as well as various surgical options including proximal and distal ligation, aneurysmectomy and aneurysm resection, are reserved for patients with significant $\mathrm{CAD}$ and ectatic changes [7, 20]. For patients with KD who had severe ischemic heart disease and underwent cardiac transplant, there has been no recurrence of coronary lesions in the transplanted heart [21].

\section{Conclusions}

Long term adverse cardiovascular outcomes are higher in ectatic infarcted related artery (EIRA) compared with non-ectatic coronary disease. It should be emphasized that coronary artery ectasia is not a benign finding. Dilated ectatic vessels predispose to various complications including thrombus formation, arterial spasm, spontaneous dissection, and MI. Percutaneous intervention and stent placement has limited role in management of EIRA given the unfavorable anatomy. Moreover, there is a lack of clear guidelines regarding the use of dual antiplatelet therapy, triple blood thinning therapy, or their optimal duration. This patient developed recurrent MI despite optimum health measures and dual antiplatelet therapy. A clear guideline on management of this condition is needed.

\section{References}

1. Eitan A, Roguin A. Coronary artery ectasia: new insights into pathophysiology, diagnosis, and treatment. Coron Artery Dis. 2016;27(5):420-428.

2. Burns JC, Kushner HI, Bastian JF, Shike H, Shimizu C, Matsubara T, Turner CL. Kawasaki disease: A brief history. Pediatrics. 2000;106(2):E27.

3. Falcini F, Capannini S, Rigante D. Kawasaki syndrome: an intriguing disease with numerous unsolved dilemmas. Pediatr Rheumatol Online J. 2011;9:17.

4. Kontopoulou T, Kontopoulos DG, Vaidakis E, Mousoulis GP. Adult Kawasaki disease in a European patient: a case report and review of the literature. J Med Case Rep. 2015;9:75. 
5. Bhagwat A, Mukhedkar S, Ekbote S, Gordon JB. Missed Kawasaki disease in childhood presenting as myocardial infarction in adults. Indian Heart J. 2015;67(4):385-388.

6. Senzaki H. Long-term outcome of Kawasaki disease. Circulation. 2008;118(25):2763-2772.

7. Mavrogeni S. Coronary artery ectasia: from diagnosis to treatment. Hellenic J Cardiol. 2010;51(2):158-163.

8. Kruger D, Stierle U, Herrmann G, Simon R, Sheikhzadeh A. Exercise-induced myocardial ischemia in isolated coronary artery ectasias and aneurysms ("dilated coronopathy"). J Am Coll Cardiol. 1999;34(5):1461-1470.

9. Lamblin N, Bauters C, Hermant X, Lablanche JM, Helbecque N, Amouyel P. Polymorphisms in the promoter regions of MMP-2, MMP-3, MMP-9 and MMP-12 genes as determinants of aneurysmal coronary artery disease. J Am Coll Cardiol. 2002;40(1):43-48.

10. Sorrell VL, Davis MJ, Bove AA. Origins of coronary artery ectasia. Lancet. 1996;347(8995):136-137.

11. Demopoulos VP, Olympios CD, Fakiolas CN, Pissimissis EG, Economides NM, Adamopoulou E, Foussas SG, et al. The natural history of aneurysmal coronary artery disease. Heart. 1997;78(2):136-141.

12. Gziut AI, Gil RJ. Coronary aneurysms. Pol Arch Med Wewn. 2008;118(12):741-746.

13. Papadakis MC, Manginas A, Cotileas P, Demopoulos V, Voudris V, Pavlides G, Foussas SG, et al. Documentation of slow coronary flow by the TIMI frame count in patients with coronary ectasia. Am J Cardiol. 2001;88(9):10301032 .

14. Ozbay Y, Akbulut M, Balin M, Kayancicek H, Baydas
A, Korkmaz H. The level of hs-CRP in coronary artery ectasia and its response to statin and angiotensin-converting enzyme inhibitor treatment. Mediators Inflamm. 2007;2007:89649.

15. Kosar F, Acikgoz N, Sahin I, Topal E, Aksoy Y, Cehreli $\mathrm{S}$. Effect of ectasia size or the ectasia ratio on the thrombosis in myocardial infarction frame count in patients with isolated coronary artery ectasia. Heart Vessels. 2005;20(5):199-202.

16. Hirapur I, Veeranna R, Agrawal N. Regurgitation of blood flow from the ectatic LAD artery as a cause of angina demonstrated during coronary angiogram. BMJ Case Rep. 2014;2014.

17. Group JCSJW. Guidelines for diagnosis and management of cardiovascular sequelae in Kawasaki disease (JCS 2013). Digest version. Circ J. 2014;78(10):2521-2562.

18. Yetkin E, Waltenberger J. Novel insights into an old controversy: is coronary artery ectasia a variant of coronary atherosclerosis? Clin Res Cardiol. 2007;96(6):331-339.

19. Sorrell VL, Davis MJ, Bove AA. Current knowledge and significance of coronary artery ectasia: a chronologic review of the literature, recommendations for treatment, possible etiologies, and future considerations. Clin Cardiol. 1998;21(3):157-160.

20. Manginas A, Cokkinos DV. Coronary artery ectasias: imaging, functional assessment and clinical implications. Eur Heart J. 2006;27(9):1026-1031.

21. Checchia PA, Pahl E, Shaddy RE, Shulman ST. Cardiac transplantation for Kawasaki disease. Pediatrics. 1997;100(4):695-699. 